\title{
Per la città e per il regno: dialettica politica e resistenza nelle rivolte palermitane del 1647
}

\author{
Daniele Palermo
}

Università di Palermo

Per la ciutat i pel regne: dialèctica política i resistència a les revoltes de Palerm del 1647

\section{Resum}

Durant les revoltes de Palerm de la primavera-estiu del I647, en les quals es van produir interessants dialèctiques polítiques, es va configurar una nova representació de les relacions de poder entre el Regne de Sicília i la Corona espanyola. En una conjuntura difícil i complexa per al Regne de Sicília i la resta del sistema espanyol, a Palerm, i a la majoria de ciutats de l'illa per on es va estendre la revolta, es van abolir les gabelle dels productes alimentaris de primera necessitat i es va permetre als exponents de l'"univers popular» l'accés al govern cívic i als seus càrrecs més alts. Tot això podria haver canviat les relacions amb la Corona: modificaria la funció del donativo i les relacions entre el monarca i les elits urbanes esdevindrien més complexes. No obstant això, el fracàs en l'intent de fixar als capitoli unes reformes vàlides per a tot el Regne acabà determinant el retorn a l'ordre anterior.

Paraules clau: Sicília, revoltes, gabelle, govern. 
Por la ciudad y por el reino: dialéctica politica y resistencia en las revueltas de Palermo de 1647

\section{Resumen}

Durante las revueltas de Palermo de la primavera-verano de I647, en las que se produjeron interesantes dialécticas políticas, se configuró una nueva representación de las relaciones de poder entre el Reino de Sicilia y la Corona española. En una coyuntura difícil y compleja para el Reino de Sicilia y el resto del sistema español, en Palermo, y en la mayoría de las ciudades de la isla por las que se extendió la revuelta, se abolieron las gabelle de los productos alimentarios de primera necesidad y se permitió a los exponentes del «universo popular» el acceso al gobierno cívico y a sus más altos cargos. Todo esto podría haber cambiado las relaciones con la Corona: se modificaría la función del donativo y las relaciones entre el soberano y las élites urbanas se volverían más complejas. Sin embargo, el fracaso al intentar fijar en los capitoli reformas válidas para el resto del Reino acabaría por determinar la restauración del orden anterior.

Palabras clave: Sicilia, revueltas, gabelle, gobierno.

For city and kingdom: political usages and resistance in the Palermo revolts of 1647

\section{Summary}

During the revolts that took place in Palermo in the spring-summer of I647, when there were interesting political dialectics, a possible new representation of the relationship between the Kingdom of Sicily and the Crown was outlined. In the context of a difficult and complex phase both of the Kingdom of Sicily and of the entire Spanish system, in Palermo and in most of the island's cities which staged revolts, the gabelle on basic necessities were abolished and exponents of the "popular universe" allowed access to the civic government, right up to its highest levels. All this could have changed relations with the Crown: the function of the donativo would have changed and the relations of the king with the urban elites would have become more complex. The failure of the attempt to establish reforms valid for the whole kingdom in the capitoli determined the restoration of the previous order.

Keywords: Sicily, revolts, gabelle, government. 
A partire dagli ultimi anni del xx secolo, l'utilizzo da parte della storiografia di concetti come "conflitto" e "fazione" nell'analisi di carattere politico delle rivolte di antico regime è testimonianza dell'attenzione per categorie nuove rispetto a quelle più tradizionali di "rivolta" e "rivoluzione". ${ }^{1}$ Negli stessi anni un importante filone di studi ha letto, in un'ottica ancor più peculiarmente politica, le rivolte europee del XVII secolo come occasioni di affermazione del diritto di resistenza contro comportamenti del sovrano considerati tirannici. ${ }^{2}$ La rivolta o la rebellio non sono mai legittime, la resistenza viene considerata o si autorappresenta come legittima; la categoria di rivolta appare dunque insufficiente a descrivere l'universo di rapporti politici che caratterizzano la società moderna. ${ }^{3}$

Prettamente politica, molto più che socio-antropologica, è la prospettiva comparativistica offertaci da Jeoffrey Parker: la scala temporale scelta è di breve periodo e la proposta interpretativa è caratterizzata da fattori quali gli effetti della pressione fiscale legata alle spese militari; il ruolo di intelectuales alienados, laici ed ecclesiastici, che stentavano a inserirsi nelle dialettiche politiche locali; le istanze di rappresentanza paritaria tra «nobili» e "popolari» nel governo delle città, soprattutto per ciò che riguarda le capitali; l'importanza attribuita alla convocazione di assemblee che stilassero programmi di riforme valide per l'intero regno; l'ampia e rapida circolazione di istanze e rivendicazioni. ${ }^{4}$

I. Tra gli altri ci si riferisce a Francesco Benigno, «Conflitto politico e conflitto sociale nell'Italia spagnola", in A. Musi, ed., Nel sistema imperiale: l'Italia spagnola, Edizioni Scientifiche Italiane, Napoli, I995, pp. II5-I46.

2. Daniele Palermo, «Percorsi storiografici sul Xvi secolo: dalla rivolta alla resistenza», Mediterranea. Ricerche storiche, 22 (201I), pp. 313-332. Ci si riferisce, tra gli altri lavori, a Angela De Benedictis, «Identità comunitarie e diritto di resistere», in P. Prodi, W. Reinhard, eds., Identità collettive tra Medioevo ed età moderna. Convegno internazionale di studio, Il Mulino, Bologna, 2002, pp. 265-294 e Angela De Benedictis, Tumulti. Moltitudini ribelli in età moderna, Il Mulino, Bologna, 20I3, in particolare pp. IOI-I6I.

3. Angela De Benedictis, «Rivolte, ribellioni, resistenze. Letture di testi e problemi storiografici», in A. Lerra, A. Musi, eds., Rivolte e rivoluzione nel Mezzogiorno d'Italia. 1547-I799, Lacaita, Manduria, 2008, pp. 297-302.

4. Geoffrey Parker, Global crisis. War, climate change and catastrophe in the seventeenth century, Yale University Press, New Haven / London, 2012. 
Le due rivolte palermitane del maggio e dell'agosto I647 sono state tradizionalmente interpretate in senso non politico. Proponendo una lettura alternativa, Francesco Benigno sottolinea come, tra le sei «rivoluzioni contemporanee» del xvir secolo oggetto dell'opera di Merriman, quella di Palermo «è stata considerata come la meno rilevante, vista tradizionalmente come un classico esempio di rivolta per la fame o, nella migliore delle ipotesi, di jacquerie urbaine». Egli attribuisce invece a questi eventi il significato, prettamente politico, di «reazioni parallele a uno stesso modello di governo monarchico che aveva assunto, soprattutto grazie alla teoria e pratica del governo straordinario e di guerra con la presenza di ministri favoriti, il volto assai tradizionale ma non per questo meno aborrito del regime tirannicom. ${ }^{5}$

Il panorama degli anni che precedettero le rivolte del I647 fu caratterizzato dal devastante impatto della guerra dei Trent'anni sull'Euro$\mathrm{pa}$, non estraneo alla difficile congiuntura economica che caratterizzava buona parte del continente; ma non solo, perché a questo si aggiungevano la crisi della privanza, che aveva raggiunto il culmine proprio all'inizio degli anni '40, e le drammatiche rivolte di Catalogna e Portogallo, ${ }^{6}$ "effetto della flessibilità che il valimiento conferiva al sistema..., rendendo in qualche modo compatibili la lealtà al sovrano e l'opposizione al suo ministro», e la lacerazione, forse una vera e propria rottura dei legami politici tra ceti dirigenti locali e grande aristocrazia castigliana. ${ }^{7}$ Questa complessa situazione gravava anche sulla Sicilia, dove le tensioni crescevano e si diffondevano, in un quadro generale che sembrava mettere a rischio l'esistenza stessa della monarchia iberica.

5. Francesco Benigno, “Fora gabelle e malogoverno”. Riflessioni sulla rivolta di Palermo", in Lerra, Musi, Rivolte e rivoluzione, pp. I9I-197. Sulle rivolte siciliane del I647, cfr. Daniele Palermo, Sicilia 1647. Voci, esempi, modelli di rivolta, Associazione Mediterranea, Palermo, 2009; Daniele Palermo, "Sicilia in rivolta», in A. Giuffrida, F. D’Avenia, D. Palermo, eds., La Sicilia del '6oo. Nuove linee di ricerca, Associazione Mediterranea, Palermo, 2012, pp. II5-I68.

6. Parker, Global crisis.

7. Francesco Benigno, L'ombra del re. Ministri e lotta politica nella Spagna del ‘6o, Marsilio, Venezia, I992, pp. I42-I45. 
Peculiare, invece, del contesto delle rivolte siciliane del I647 è senza dubbio il dualismo tra Palermo e Messina: una vera e propria rivalità «che si alimentava di ragioni che andavano al di là del pur così diffuso contenzioso municipalistico». ${ }^{8}$ Gli eventi dell'isola e ogni disegno di trasformazione dei rapporti con la Corona si incrociano e si intrecciano con quella particolare rappresentazione dell'articolazione policentrica del potere e della fedeltà che caratterizzava il Regno di Sicilia e che Benigno ha indicato come la «questione della capitale».

Due città si contenderanno a lungo il ruolo di capitale: Palermo, caratterizzata dalle dialettiche politiche dell'aristocrazia e degli operatori economici attorno ai poteri del Regno, in una talvolta palese rappresentazione di fedeltà al sovrano, e con un Senato teoricamente aperto ai non nobili ma sempre composto da grandi aristocratici del Regno; Messina, la cui identità civica era caratterizzata da una fedeltà alla corona di stampo pattizio e quasi repubblicano e dalla resistenza a ogni forma di accentramento e il cui Senato era composto anche da "giurati cittadini».

Il dualismo tra i due centri urbani era destinato a emergere in maniera ancor più radicale durante le rivolte del I647, allorché la città di Messina non solo avrebbe dichiarato ripetutamente la propria fedeltà al sovrano, stigmatizzando il grave comportamento dei Palermitani, ma si sarebbe adoperata anche con proprie forze militari e con l'impegno diretto dei propri ufficiali nelle operazioni di repressione e di controllo dell'ordine pubblico nel suo comprensorio. Il medesimo atteggiamento sarebbe stato adottato dalla città di Palermo, allorché, nel I674, a rivoltarsi sarebbero stati i Messinesi. ${ }^{9}$

Infine, in una seppur sintetica lettura politica delle rivolte siciliane, appare indispensabile tenere in considerazione la rappresentazione, ar-

8. Francesco Benigno, «La questione della capitale: lotta politica e rappresentanza degli interessi nella Sicilia del '60o", Società e storia, 47 (1990), pp. 27-59; Ennio Igor Mineo, «Sicilia urbana», in F. Benigno; C. Torrisi, eds., Rappresentazioni e immagini della Sicilia tra storia e storiografia, Sciascia, Caltanissetta / Roma, 2003, pp. 19-39.

9. Luis Antonio Ribot García, La Monarquía de España y la guerra de Mesina (I674-I687), Actas, Madrid, 2002; cfr. anche Salvatore BotTARi, Post res perditas. Messina I678-17I3, Sfamemi, Messina, 2005. 
tificiale e costruita, del rapporto tra il Regno di Sicilia e la Corona: in conseguenza della Guerra del Vespro, il Regno tramite i suoi baroni si sarebbe liberamente donato a Pietro III di Aragona. Simbolo concreto di questo, ma ugualmente frutto di una rappresentazione, è il donativo concesso periodicamente al sovrano dal Parlamento. ${ }^{10}$

Questa rappresentazione complessiva entra in crisi nei decenni immediatamente precedenti alla rivolta allorché i mattoncini alla base della fedeltà di un Regno che si considerava liberamente donato crollano. Entrano infatti in una crisi quasi irreversibile le università demaniali, poteri fedeli alla Corona e al viceré, che non riescono a far fronte alle rate dei donativi, determinando un complessivo indebitamento del Regno e una crisi di rapporti col viceré, loro tradizionale interlocutore e sostegno. ${ }^{11}$ Il dissesto finanziario delle città e la pesante pressione fiscale erano ritenuti tra le più gravi cause di crisi anche dai contemporanei. ${ }^{12}$

A Palermo, in modi e tempi forse non imprevisti, il 20 maggio del I647, protestando per la riduzione del peso delle forme di pane stabilita qualche giorno prima, effetto della crisi alimentare in corso, una «folla», composta da "muchachos, mugeres y gente vaxa», occupò le piazze e diede l'assalto ai palazzi pubblici e alle abitazioni degli ufficiali delle città e del Regno, ritenuti responsabili dell'accaduto. ${ }^{13}$ Come scrive Francesco Benigno,

Io. Una rassegna delle rappresentazioni del rapporto tra il Regno di Sicilia e la Corona in Pietro Corrao, "La Sicilia provincia», in Benigno, Torrisi, Rappresentazioni e immagini, pp. 4I-58.

II. Giuseppe Giarrizzo, «La Sicilia dal Cinquecento all'Unità d'Italia», in V. D’Alessandro, G. Giarrizzo, eds., La Sicilia dal Vespro all'Unità, UTET, Torino, pp. 280-290, 297-298.

I2. Si veda Don Cesare Moncada, principe di Calvaruso, ai consiglieri del viceré, Caltanissetta, Io giugno I647, Archivio di Stato di Palermo (ASP), Real Segreteria-Incartamenti (RS-I), leg. I653, f. 206 r-v.

13. Consulta del Consiglio d'Italia del 17 giugno I647, Archivo General de Simancas (AGS), Secreterías Provinciales-Sicilia (SP-S), leg. I444, fogli non numerati; cfr. anche Don Luis De Los Cameros a Filippo IV, Palermo, 25 maggio I647, ivi; Gli inquisitori Lopez e Trasmiera a Filippo IV, Palermo, 23 maggio I647, Archivo Historico Nacional (AHN), Inquisición, leg. I698, fogli non numerati. 
[...] anche nella primissima fase del conflitto, il terreno di confronto politico che si apre in città oltrepassa rapidamente la questione, vitale ma ristretta [...] del peso del pane. Superato un primo momento, in cui la carestia appariva naturalmente il castigo divino per i peccati della città, con la conseguente coda abituale di penitenze collettive e di processione di flagellanti, l'emergere di una responsabilità umana (e cioè politica) del caro-pane divenne rapidamente opinione comune. ${ }^{14}$

Il viceré in carica Pietro Faxardo Zuñiga e Requenses, marchese di Los Vélez, si trovò a gestire una grave emergenza. Egli era stato coinvolto in uno degli episodi chiave della crisi della privanza: aveva comandato l'armata incaricata di reprimere la rivolta catalana che era stata sconfitta nel 1640 al Montjuic. ${ }^{15}$ Aveva ricevuto la nomina il I8 dicembre I643, pochi mesi dopo l'allontanamento dell'Olivares dalla carica di valido, ed era succeduto al conte di Modica Giovanni Enriquez de Cabrera, leader di una delle fazioni nobiliari che si opponevano risolutamente all'Olivares e alle sue politiche. ${ }^{16}$ Nonostante l'incapacità da lui dimostrata nell'affrontare il grave disavanzo delle finanze del Regno, alla vigilia dell'ondata di rivolte della primavera-estate del I647, lo stile di governo del Los Vélez, ormai alla fine del suo mandato, era giudicato prudente dal Consiglio d'Italia, che tuttavia riteneva preoccupanti le continue voci sui suoi possibili successori. Si credeva infatti che una transizione eccessivamente lunga indebolisse l'azione e il prestigio del viceré in carica e degli ufficiali spagnoli. ${ }^{17}$ In un modello di sovranità figlio dello "stile aragonese" e in un orizzonte di "monarchia composita”, i viceré di Sicilia si trovavano all'incrocio di equilibri, squilibri e

14. Benigno, "Fora gabelle e malo governo", pp. 197-198.

I5. John H. ElLiott, The revolt of the Catalans. A study in the Declin of the Spain, 1598-1640, Cambridge University Press, Cambridge, I963.

16. Giovanni Evangelista Di Blasi, Storia cronologica dé Viceré, Luogotenenti e Presidenti del Regno di Sicilia, 4 vols., Edizioni della Regione siciliana, Palermo, I974, vol. III, pp. I29-I35; BENIGNo, L'ombra del re, p. I39.

I7. Consulta del Consiglio d'Italia, AGS, SP-S, leg. I444, documento non datato ma, con ogni probabilità, dell'inverno del I646, fogli non numerati. 
tensioni tra la Corona, la fazione dominante a corte, l'aristocrazia siciliana, i poteri del Regno, i poteri della capitale - comprese le maestranze, unica possibilità di partecipazione alla vita politica cittadina da parte dei membri dell' universo popolare ${ }^{18}$ — e i poteri della città di Messina.

Tra gli elementi che prepararono l'emergenza insurrezionale e che devono essere considerati a questa strettamente legati vi è proprio la condizione del viceré: stanco, a fine mandato e che chiedeva di recarsi in Spagna anzitempo. Altri fattori devono però essere citati: la crisi politica determinata da divisioni nell'élite del Regno, originate dal tentativo dell'antica aristocrazia nel Parlamento del I642 di alleggerire in modo unilaterale il proprio debito tramite la riduzione degli interessi - dallo scontro emerse la possibilità che si formasse una nuova élite intercetuale formata da coloro che lucravano sul debito pubblico e su quello privato; la crisi finanziaria il cui sintomo più grave era l'incapacità delle città di soddisfare le «tande» del donativo e il tentativo delle maestranze di partecipare ai più alti livelli al governo delle città. I sodalizi degli artigiani saranno protagonisti delle difficili settimane della primavera-estate del I647, anche per l'importante ruolo che detenevano: controllavano gli approvvigionamenti e detenevano una importante forza all'interno del Consiglio Civico. ${ }^{19}$

Sulla base di una sorta di copione che accomuna la gran parte delle rivolte urbane di antico regime, un vero e proprio "sapere sociale", 20

i8. Sulle maestranze a Palermo, cfr. Gabriella Lombardo, «Tra politica ed economia: le corporazioni di mestiere nella Sicilia moderna», in M. Meriggi, A. Pastore, eds., Le regole dei mestieri e delle professioni. Secoli XV-XIX, Franco Angeli, Milano, 2000, pp. 326-345; Valentina Vigiano, «I «mezzani» nella Palermo della prima metà del Cinquecento: norme, pratiche, modelli aggregativi e reti fiduciarie», ibidem, pp. 346-363; Francesco Luigi Oddo, Le maestranze di Palermo: aspetti di vita politico-culturale. Secc. XII-XIX, Accademia Nazionale di Scienze, Lettere e Arti, Palermo, I99I; F. L. Oddo, ed., Statuti della maestranze di Palermo nei secoli XV-XVIII, Istituto di storia del Vallo di Mazara, Mazara del Vallo, i99I.

19. Daniele Palermo, Sicilia I647. Voci, esempi, modelli di rivolta, Associazione Mediterranea, Palermo, 2009, pp. 23-53.

20. Arlette Farge, Jacques Revel, La logica della folla. Rapimenti di bambini nella Parigi del I750, Laterza, Roma / Bari, I989, pp. 4-5. 
dunque, il 20 maggio I647 una gran folla, all'interno della quale nel corso di alcune ore appare in modo sempre più palese il ruolo delle maestranze - nelle settimane precedenti "brodo di coltura" delle rivendicazioni —, attaccò i luoghi del potere cittadino, come il palazzo del pretore, e il palazzo del viceré. Il potere cittadino in realtà era legato in qualche modo alla Corona e agli equilibri del "sistema imperiale": pretore e capitano erano nominati dal sovrano tra i grandi aristocratici del Regno. ${ }^{21}$

Da una folla prima indifferenziata, poi sempre più identificabile con le maestranze, cominciano a emergere in modo sempre più chiaro le rivendicazioni: una diversa gestione delle risorse alimentari, più favorevole alla capitale; la soppressione o il forte ridimensionamento delle gabelle sui generi di prima necessità e, all'inizio sottotraccia, ma non meno importante delle precedenti, l'introduzione delle figure dei giurati popolari all'interno del Senato di Palermo e dunque la cessazione del monopolio su questa istituzione da parte della grande aristocrazia del Regno.

La rivolta crea anche una sorta di "modello insurrezionale" - costituito da azioni consuete, prodotto del "sapere sociale" di cui dicevamo, e nuove rivendicazioni — che si diffonde nella quasi totalità del Regno; fa eccezione l'area di influenza della città di Messina, dove questa fungerà da tutrice dell'ordine e si rappresenterà al sovrano come fedelissima e come nuova e sicura capitale in opposizione a una Palermo rappresentata come ribelle e come luogo in cui è impossibile svolgere le funzioni di governo del Regno. ${ }^{22}$

La rivolta di Palermo del maggio I647 — quella di agosto può essere considerata frutto di squilibri e tensioni politiche conseguenti alla rivolta di maggio - , dunque, genera una possibile diversa rappresentazione dei rapporti tra il Regno di Sicilia e la Corona, capace potenzialmente di sostituire quella in crisi, e questo nell'ambito di una concezione della fedeltà che nel "sistema spagnolo" consentiva la coesistenza della lealtà

2I. Sulle istituzioni della capitale, cfr. Valentina Vigiano, L'esercizio della politica. La città di Palermo nel Cinquecento, Viella, Roma, 2004.

22. Palermo, Sicilia 1674, pp. 55-74. 
al sovrano e della possibilità di ridefinire assetti politici e sociali, anche attraverso la rivolta. ${ }^{23}$

Il primo versante di questa diversa rappresentazione sarebbe stato basato sull'assenza di gabelle, dunque di imposte indirette sui generi di prima necessità, e avrebbe determinato l'esigenza di cercare imposizioni alternative, pena l'impossibilità di pagare i donativi, prospettiva concretissima qualora le città demaniali, su cui ricadeva gran parte del peso dei donativi ordinari e straordinari, avessero dovuto rinunciare allo strumento principale e talvolta unico per reperire il denaro. Anche se mai a livello teorico, entrava in concreta crisi lo strumento del donativo, politicamente rappresentato come la contribuzione volontaria di un Regno che liberamente si è donato al sovrano; si trattava di un canale simbolico e a volte concreto di fedeltà al sovrano non solo da parte del Regno ma anche delle città demaniali, e tutte le città più importanti, ad eccezione di Modica, erano demaniali. ${ }^{24}$

Il secondo versante di questa diversa rappresentazione dei rapporti con la Corona è il disegno di introdurre giurati popolari ove i Senati/ Giurazie fossero "chiusi», riservati alla nobiltà; ciò avrebbe determinato, almeno temporaneamente, nuovi equilibri politici soprattutto a Palermo, dove sarebbe entrato in crisi un canale che, attraverso l'assunzione del monopolio sul Senato, legava la grande aristocrazia al viceré e al sovrano. L'istituzione dei giurati popolari, elemento fondamentale di un modello basato su una piena attuazione della dialettica tra "universo popolare" e "universo nobiliare", era stata, secondo Koenigsberger, frutto dell'interazione nei mesi precedenti tra maestranze e «intellettuali della classe media», come Francesco Baronio. ${ }^{25}$

23. Xavier GiL, «The good law of a vassal: fidelity, obedience and obligation in Habsburg Spain", in J. Arrieta, J. H. Elliott, eds., Forms of union: the British and Spanish monarchies in the seventeenth and eighteenth centuries, Cuadernos de la Revista Internacional de Estudios Vascos, 5 (2009), pp. 83-106.

24. Sul donativo, cfr. Rossella Cancila, Fisco, ricchezza, comunità nella Sicilia del Cinquecento, Istituto Storico Italiano per l'Età Moderna e Contemporanea, Roma, 200I.

25. Helmut Georg Koenigsberger, "The revolt of Palermo in I647", The Cambridge Historical Journal, 8 (1946), p. I4I. Francesco Benigno ritiene non sufficienti gli 
Sin dalle prime ore della rivolta emerge l'importante ruolo dei mediatori, efficace canale di analisi delle implicazioni politiche di una situazione di grave crisi. Si tratta di religiosi, come il gesuita Salvago e il teatino Giardina, che incanalavano le richieste della folla verso istanze che non sovvertissero l'ordine sociale, sostanzialmente quelle delle maestranze; di aristocratici dal comportamento ambiguo che probabilmente cercavano di approfittare della resistenza al potere cittadino e a quello viceregio per accrescere il proprio ruolo nel teatro politico di Palermo e del Regno; di uomini legati alla Corona ma allo stesso tempo inseriti nella dialettica dei poteri del Regno come l'arcivescovo di Palermo Fernando de Andrade e l'arcivescovo di Monreale Juan de Torresilla; di personaggi apparentemente in stretto rapporto col viceré, come il giudice della Regia Monarchia Luis de Los Cameros; di individui apparentemente legati alla Corona e potenzialmente slegati dai poteri del Regno, come gli inquisitori Lopez e soprattutto Trasmiera. ${ }^{26}$

Un filo costante accomuna le settimane tra la rivolta di maggio e i primi giorni di luglio, e si tratta della presenza continua degli uomini dell'inquisizione in funzione di mediatori: Lopez e Trasmiera, riferendo al sovrano, parleranno di sé stessi come gli unici a mantenere una palese e indiscussa fedeltà nei suoi confronti in quei difficili momenti. ${ }^{27}$

elementi — solo la testimonianza del «polemista messinese» Reina — per potere affermare che alla base della richiesta di istituzione dei giurati popolari vi possa essere «l'azione di intellettuali [...] che avevano fatto dell'esaltazione municipalistica (ma anche della polemica antimessinese) la loro bandiera" (BEnigno, "Fora gabelle e malo governo", pp. 20I-203).

26. Consulta del Consiglio d'Italia del 17 giugno I647, AGS, SP-S, leg. I444, fogli non numerati; cfr. anche Gli inquisitori Lopez e Trasmiera a Filippo IV, Palermo, 23 maggio I647, AHN, Inquisición, leg. I698, fogli non numerati; Don Luis De Los Cameros a Filippo IV, Palermo, 25 maggio I647, AGS, SP-S, leg. I444, fogli non numerati. Cfr. anche Palermo, Sicilia I647, pp. 55-86.

27. Manuel Rivero Rodríguez, «Técnica de un golpe de Estado: el inquisidor Garcia de Trasmiera en la revuelta siciliana de I647", in F. J. Aranda Perez, ed., La declinación de la Monarquía Hispánica en el siglo XVII, Ediciones de la Universidad de Castilla-La Mancha, Cuenca, 2004, pp. I29-I53. 
Ottenute il 2I maggio le concessioni dal viceré - migliori approvvigionamenti per la città, l'abolizione della gabelle e l'istituzione di due posti di giurato popolare nel Senato di Palermo - le maestranze mutarono la loro posizione nel teatro politico cittadino e, lasciato il "fronte popolare", entrarono a far parte del "partito dell'ordine" e divennero garanti e responsabili delle scelte conseguenti diversi equilibri sociali e politici.

Il ruolo delle maestranze era divenuto importante e delicato: gli artigiani in armi sorvegliavano la città in un momento in cui il controllo dell'ordine pubblico della capitale era vitale per l'esistenza del Regno; avevano la possibilità di rendere reali una società e una economia non gravate da onerose imposte sui generi di prima necessità; erano pienamente coinvolte nel governo della città al suo livello più alto. Proprio alla capacità delle maestranze di operare scelte adeguate è legata la possibilità di sopravvivenza di una diversa rappresentazione dei rapporti tra il Regno di Sicilia e la Corona. In primo luogo questa si sostanziava precisamente nell'assenza di uno stretto vincolo tra gabelle e donativo; sarebbe spettato proprio alle maestranze di Palermo l'arduo compito di trovare cespiti per garantire il pagamento delle «tande» ed evitare l'interruzione di un canale continuo di flusso finanziario verso la Corona. Il tentativo si rivelerà inutile e infruttuoso.

Questa diversa rappresentazione era caratterizzata in secondo luogo dalla presenza anche di due giurati popolari, quindi dell'intero universo politico cittadino, nel Senato di Palermo, e dunque dalla fine del rapporto duplice ed esclusivo tra re e pretore, da una parte, e viceré e senatori dall'altra, e da relazioni dunque più complesse e complicate tra la Corona e un'élite cittadina in procinto di divenire intercetuale. Questa apertura del Senato cittadino sarebbe potuta divenire realtà solo se le maestranze fossero riuscite a scegliere come giurati popolari uomini autorevoli, ampiamente graditi, capaci di mediazione e in grado di rappresentare gli interessi della città e dunque, in quei momenti di crisi finanziaria e alimentare, di reperire capitali e derrate per sopperire alle sue necessità; in questo l'operato dei gruppi dirigenti delle maestranze fu coronato da successo.

Ovviamente altre forze giocano, seppur in modo incerto e non lineare, sulla scena politica e abbastanza identificabile sembra l'azione 
della grande aristocrazia che cerca di sottrarre alle maestranze il rilevante potere da loro conquistato e di preparare il ripristino dell'ordine, diffondendo la voce di un prossimo trasferimento del viceré e dei tribunali del Regno a Messina, così da determinare ulteriori divisioni nel fronte che aveva dato vita alla rivolta. Ciò che veniva ventilato avrebbe costituito una sciagura, poiché sulla presenza delle strutture politico-amministrative del Regno e su quella collaterale della grande aristocrazia ruotava gran parte dell'economia della città.

In un'analisi della situazione politica relativa ai giorni compresi tra la conclusione della prima rivolta e il 15 agosto, in cui prenderà avvio una seconda drammatica rivolta, sono riscontrabili alcune linee di continuità: emerge l'inadeguatezza delle scelte effettuate dai gruppi dirigenti delle maestranze relativamente alle imposte alternative individuate in sostituzione di quelle sui generi di prima necessità, poiché risultano sostanzialmente inesigibili; prosegue dunque la crisi finanziaria di Palermo e, poiché le altre città l'hanno seguita nell'abolizione delle gabelle, dell'intero Regno; si verifica una frattura, che diviene sempre più ampia tra maestranze ricche e potenti, che avevano beneficiato delle concessioni del viceré, e altri sodalizi o gruppi non formalmente organizzati rimasti ai margini dell'arena politica cittadina; infine, si accresce l'instabilità politica, rappresentata da membri dell'élite del Regno come drammatica conseguenza di una lenta transizione verso l'arrivo di un nuovo viceré. ${ }^{28}$

Negli ultimi giorni di maggio, poco dopo l'inizio dei tumulti di Palermo, anche Catania fu teatro di una grave rivolta e si poté parlare di una vera e propria ondata insurrezionale, che si irradiava nell'isola da due epicentri, come la capitale e la città etnea, da cui venivano veicolati messaggi precisi riguardanti soprattutto le istanze avanzate dai rivoltosi. Tumulti si verificarono nelle principali città del Regno, ad eccezione di Messina, e di particolare gravità furono quelli di Girgenti, Bronte e Caltanissetta. ${ }^{29}$

28. Palermo, Sicilia I647, pp. 55-86.

29. Una cospicua documentazione sulle rivolte siciliane in ASP, RS-I, legg. I653 e 1654 . 
Il Is agosto ebbe inizio una nuova rivolta sostanzialmente guidata proprio da quei soggetti collettivi - maestranze come quella dei conciatori o gruppi non formalmente organizzati, come quello dei pescatori che non avevano tratto vantaggi dagli eventi di maggio. ${ }^{30} \mathrm{~A}$ differenza della rivolta di maggio, comparve sulla scena un capopopolo ben individuabile e, a detta di Francesco Benigno, dai caratteri masanielliani: si trattava di Giuseppe D’Alesi.

Masanielliana è la leggenda della sua elezione a capopopolo a sorte in un'osteria. Masanielliana la tragedia del suo meteorico consenso popolare, rapidamente conquistato e altrettanto rapidamente perduto: in ragione delle sue scelte ma anche a causa delle arti melliflue di chi lo voleva mettere in cattiva luce per farlo perdere. Masanielliana, anche se con qualche significativa variante, è la sua fine drammatica. E tuttavia vi è una differenza fondamentale tra i due personaggi. D'Alesi non riesce mai a raggiungere quella autorità carismatica assoluta che costituisce il tratto pregnante di Masaniello [...] egli non assurge, come Masaniello, a simbolo dell'identità popolare [...] Soprattutto la sua presenza non arriva a sciogliere o a mascherare l'autonomia dei vari gruppi di maestranze che compongono il popolo e che mantengono, a Palermo più che a Napoli, una propria autonomia e fisionomia. ${ }^{31}$

Si determina già dalle prime ore della rivolta una singolare situazione nell'esercizio del potere, infatti il viceré Los Vélez si rifugia su una nave che getterà l'ancora di fronte alla borgata marinara dell'Arenella. È dunque difficile comprendere chi per alcuni giorni gestirà il potere in città.

L'inizio della seconda rivolta comportò un'accentuata dialettica tra resistenza e fedeltà. Appare evidente la resistenza e ne è testimonianza la fuga da Palermo del viceré, che pure vi restava vicino, era raggiungibile da chi voleva comunicare con lui, vedeva la città e quasi ne respirava l'aria. Altrettanto palesi appaiono i segni di fedeltà al sovrano della ca-

30. Daniele Palermo, «Sicilia in rivolta», in A. Giuffrida, F. D’Avenia, D. Palermo, eds., La Sicilia del '6oo. Nuove linee di ricerca, Associazione Mediterranea, Palermo, 20I2, pp. I49-I5O.

3I. Benigno, "Fora gabelle e malo governo", pp. 206-208. 
pitale e del Regno espressi nel teatro pubblico. Una vera e propria cerimonia, promossa anche dal capopopolo, si svolse il I6 agosto: nei "Quattro cantoneri», uno dei teatri della cerimonialità urbana di Palermo, fu posto il ritratto del sovrano sovrastato da un baldacchino mentre le maestranze prestavano la loro "guardia di onore». Il capopopolo era sempre accompagnato da uno stendardo con le armi del sovrano, che sarebbe diventato addirittura una caratteristica della sua immagine.

Durante la rivolta di agosto, vi fu un vero e proprio momento di snodo, in cui avrebbe potuto acquisire stabilità la diversa rappresentazione dei rapporti della Corona che rendeva più instabile il donativo e moltiplicava i canali di contatto con le élite urbane. In una lunga serie di adunanze vennero formulati i capitoli: un vero e proprio programma "costituzionale" per l'intero Regno, elaborato dai consoli delle maestranze con l'apporto di mediatori e consiglieri autorevoli, in cui si potrebbe riscontrare quella rappresentazione di cui stiamo parlando.

Alle adunanze parteciparono: il giudice della Regia monarchia Los Cameros, vicino al viceré; l'inquisitore Trasmiera che per le funzioni che ricopriva era figura vicina al sovrano; e grandi aristocratici come i principi di Trabia e di Scordia. L'esigenza di elaborare i capitoli fu giustificata, pressoché da tutte le parti coinvolte, con la necessità di evitare che viceré e tribunali si trasferissero a Messina.

Nel corso dei lavori, spesso convulsi, risultò determinante l'intervento del giurista Meroldo che convinse l'assise a mutare la natura di quanto sarebbe stato approvato: non si sarebbe più trattato di capitoli tra viceré e capopopolo ma di suppliche del Senato al viceré. Lo si sarebbe pregato di confermare quanto in suo potere e, dove non competente, di intercedere col sovrano; i capitoli divenivano così revocabili.

Si trattava di un lungo elenco di richieste, tra le quali: il ripristino dei privilegi del Regno, che erano il tratto distintivo di una rapporto con la Corona di tipo aragonese; un maggior numero di uffici riservati ai siciliani e ai palermitani; l'abolizione delle gabelle in tutto il Regno ma non delle «tande» dei donativi a carico di ogni singola università; la perpetuità dei giurati popolari — e questa era la più delicata delle richieste, infatti, per esempio, a Palermo i rappresentanti "popolari" avrebbero costituito il 50\% del Senato. 
Approvati i capitoli, l'Inquisizione, soprattutto attraverso Trasmiera, organizzò la repressione nella quale furono impiegati molti uomini di maestranze scontente o gruppi di mestiere, come i pescatori, rimasti ai margini della scena politica cittadina o forniti dagli aristocratici. Si giunse dunque, a una sola settimana dall'inizio della rivolta, il 22 agosto, all'uccisione di Giuseppe D'Alesi; seguì la revoca dei capitoli. ${ }^{32}$

Dopo l'improvvisa morte del viceré Los Vélez, giunse in Sicilia il nuovo presidente del Regno cardinale Trivulzio. Nel timore che si ripetesse ciò che stava avvenendo nel Regno di Napoli, questi adottò una politica cauta e complessa: operò la repressione nelle periferie e cercò invece la collaborazione delle maestranze nella capitale. Queste ultime parteciparono alla "Deputazione di nuove gabelle», il cui compito era la reimposizione dei gravami sui generi di prima necessità e, dopo breve tempo, sostanzialmente avallarono l'abolizione dei giurati popolari. Con cautela, ma con decisione e fermezza, il viceré Trivulzio ripristinò l'ordine sociale, politico e "costituzionale". ${ }^{33}$

\section{Appendice}

Contenuto dei capitoli confermati dal viceré Los Vélez il 23 agosto $1647^{34}$

I. indulto generale per tutti i cittadini del Regno;

2. conferma per un altro triennio del viceré Los Vélez;

3. carica di castellano da attribuire esclusivamente a cittadini palermitani «nativi»;

4. attività di macellazione monopolio esclusivo del macello della città; 5. riduzione dei «terraggi»;

32. Palermo, Sicilia in rivolta, pp. I55-I56.

33. Giarrizzo, La Sicilia, pp. 319-320.

34. Il testo integrale dei capitoli è pubblicato in Isidoro La Lumia, Giuseppe D'Alesi o la rivolta di Palermo nel 1647, Edizioni della Regione Siciliana, Palermo, I969, pp. I27-I33. 
6. dilazione di sei anni per i «borgesi» per l'arbitrio «tantum et dumtaxat»;

7. rimozione dei maestri razionali del Tribunale del real patrimonio in carica e obbligo della nomina di almeno tre palermitani su sei posti di maestro razionale;

8. abolizione della confisca dei beni per i "prosecuti»;

9. riduzione dei termini di custodia nei "dammusi» per i testimoni;

Io. ripristino di tutti i "privilegi» del Regno concessi da Pietro d'Aragona e da tutti i suoi successori sul trono di Sicilia;

II. abolizione delle gabelle in tutto il Regno, ad eccezione della regia dogana e della tande regie;

I2. reincorporazione nel demanio di città e terre vendute a privati;

I3. obbligo per gli ufficiali della città di Palermo di rendere conto del proprio operato a un'autorità appositamente nominata;

I4. obbligo per i debitori della città di versare quanto dovuto;

I5. nomina da parte del viceré di un sindacatore per esaminare l'operato dei titolari di uffici biennali;

I6. destinazione alle opere di difesa della città di una somma di denaro non inferiore a Iooo onze l'anno;

I7. Senato di Palermo composto da tre giurati nobili e tre "popolari», esclusivamente "cittadini nativi»;

I8. indicazione della chiesa di San Giuseppe come sede perpetua per l'elezione dei giurati popolari;

19. apertura di «studi publici di tutte le professioni»;

20. pagamento dei salari dei soldati non appena vi fosse la disponibilità finanziaria;

2I. disposizioni per le cause nelle quali si trovassero coinvolti giudici della Regia Corte;

22. tutti gli «offici» della città riservati a palermitani «nativi»;

23. disposizioni riguardanti «sbirri, algozirii, regi porteri e provisionati»; 24. attribuzione della cariche di deputato di piazza e maestro notario della città esclusivamente ad appartenenti alle maestranze;

25. «che circa il total giustamento della città e suggiugatarii si debia trattare e agiustare quanto prima»;

26. concessione degli «offici» del Regno solo a «regnicoli»; 
27. disposizioni sulla valutazione dei beni;

28. concessione dei «benefici ecclesiastici» del Regno solo a «regnicoli»; 29. concessione dei «benefici ecclesiastici» della città di Palermo solo a "panormitani nativi»;

30. riduzione delle "gabelle già fatte delle terre del seminerio";

3I. "che circa la variatione delli fori se alcuno n'havesse due, uno nativo e l'altro dativo, il dativo non vaglia ma debba esser convenuto nel nativo con tutto che lo renuntiasse»;

32. indivisibilità del Regno;

33. divieto di ricoprire uffici per i "prosecuti» di furto;

34. divieto di macellazione di «vitelli e vacchi lavorativi»;

35. disposizioni sull'unità di misura da usare per le terre da seminare; 36. divieto di fissare la «tratta» a più di 36 tarì per salma di grano;

37. disposizioni sulle «cessioni di raggione»;

38. limitazioni per i procedimenti del fisco contro cittadini palermitani; 39. immediata liberazione dei «forzati» non appena scontata la pena; 40. biennalità dell'ufficio di "protomedico» e sua assegnazione solo per merito;

4I. obbligo della presenza di uno dei giurati «cittadini» «nel ricevere li formenti di questa cità»;

42. garanzie per gabelloti e «inquilini»;

43. "disterro» di Silvestro Randelli;

44. abolizione della gabella sui "pesci minuti»;

45. concessione di alcune dilazioni fiscali a «tutti li poveri»;

46. impegno a emanare disposizioni circa l'abbreviamento dei tempi delle «liti»;

47. disposizioni sull'ufficio di "pesatore della seta di questa città»; 48. riduzione degli interessi per il donativo di 65.000 scudi del I644; 49. disposizioni circa le «deputazioni». 